PROCEEDINGS OF THE

AMERICAN MATHEMATICAL SOCIETY

Volume 137, Number 9, September 2009, Pages 3025-3035

S 0002-9939(09)09871-2

Article electronically published on February 11, 2009

\title{
ASYMPTOTIC BEHAVIOR OF INDIVIDUAL ORBITS OF DISCRETE SYSTEMS
}

\author{
NGUYEN VAN MINH
}

(Communicated by Nigel J. Kalton)

\begin{abstract}
We consider the asymptotic behavior of bounded solutions of the difference equations of the form $x(n+1)=B x(n)+y(n)$ in a Banach space $\mathbb{X}$, where $n=1,2, \ldots, B$ is a linear continuous operator in $\mathbb{X}$, and $(y(n))$ is a sequence in $\mathbb{X}$ converging to 0 as $n \rightarrow \infty$. An obtained result with an elementary proof says that if $\sigma(B) \cap\{|z|=1\} \subset\{1\}$, then every bounded solution $x(n)$ has the property that $\lim _{n \rightarrow \infty}(x(n+1)-x(n))=0$. This result extends a theorem due to Katznelson-Tzafriri. Moreover, the techniques of the proof are furthered to study the individual stability of solutions of the discrete system. A discussion on further extensions is also given.
\end{abstract}

\section{INTRODUCTION, NOTATION AND PRELIMINARIES}

Suppose that $T$ is a power-bounded linear continuous operator in a given complex Banach space $\mathbb{X}$, i.e., $\sup _{n \in \mathbb{N}}\left\|T^{n}\right\|<\infty$. In [12, Theorem 1] it is proven that $\lim _{n \rightarrow \infty}\left\|T^{n+1}-T^{n}\right\|=0$ if $\sigma(T) \cap\{|z|=1\} \subset\{1\}$. As noted in 20, this assertion is actually equivalent to a little weaker one that for each $x_{0} \in \mathbb{X}, \lim _{n \rightarrow \infty} \| T^{n+1} x_{0}-$ $T^{n} x_{0} \|=0$ if $\sigma(T) \cap\{|z|=1\} \subset\{1\}$. An elegant proof of this assertion, which we refer to as the Katznelson-Tzafriri Theorem, was given in [19. There are numerous works on extensions and applications of this result, of which to name a few the reader is referred to e.g. [1, 44, 5], 6] [7, 8], 10, 11, 13, 14, 16], 17, 19], and their references.

It is the primary purpose of this paper to extend the Katznelson-Tzafriri Theorem to difference equations of the form

$$
x(n+1)=B x(n)+y(n), \quad x(n) \in \mathbb{X}, n \in \mathbb{N},
$$

where $x(n) \in \mathbb{X}, B$ is a linear continuous operator acting in $\mathbb{X}$ that is not necessarily assumed to be power-bounded, and $y(n) \in \mathbb{X}$ is a sequence satisfying $\lim _{n \rightarrow \infty} y(n)=$ 0 . Our main result is Theorem 2.1, which is proven by an elementary method which can be furthered to study the stability of individual solutions of (1.1). A Tauberian theorem (Theorem [2.8) is stated and then used to prove Theorem 2.10 on the

Received by the editors November 3, 2008.

2000 Mathematics Subject Classification. Primary 47D06; Secondary 47A35, 39A11.

Key words and phrases. Katznelson-Tzafriri Theorem, discrete system, individual orbit, stability, asymptotically almost periodic.

The author is grateful to the anonymous referee for carefully reading the manuscript and for pointing out several inaccuracies and for making suggestions to improve the presentation of this paper. 
asymptotical stability of individual solutions of (1.1). This result may be seen as the discrete version of several results in [3, 17, 13, 15], and it complements a result on the strong stability of solutions in 21]. For a more complete account of results and methods in this direction the reader is referred to [4, 8, 17].

In this paper, we will use the following notation: $\mathbb{N}=\{1,2, \cdots\}, \mathbb{Z}$ is the set of all integers, $\mathbb{R}$ is the set of reals, $\mathbb{C}$ is the complex plane with $\Re z$ denoting the real part of $z \in \mathbb{C}$, and $\mathbb{X}$ is a given complex Banach space. A sequence in $\mathbb{X}$ will be denoted by $(x(n))_{n=1}^{\infty}$, or simply by $(x(n))$, and the spaces of sequences

$$
\begin{aligned}
l^{\infty}(\mathbb{X}) & :=\left\{(x(n)) \subset \mathbb{X} \mid \sup _{n \in \mathbb{N}}\|x(n)\|<\infty\right\} \\
c_{0} & :=\left\{(x(n)) \subset \mathbb{X} \mid \lim _{n \rightarrow \infty} x(n)=0\right\}
\end{aligned}
$$

are equipped with the sup-norm. The shift operator $S$ acts in $l^{\infty}(\mathbb{X})$ as follows:

$$
S x(n)=x(n+1), \quad n \in \mathbb{N}, x \in l^{\infty}(\mathbb{X}) .
$$

In this paper, for a complex Banach space $\mathbb{X}$, the space of all bounded linear operators acting in $\mathbb{X}$ is denoted by $L(\mathbb{X}) ; \rho(B), \sigma(B), \operatorname{R} \sigma(B), \operatorname{Ran}(B)$ denote the resolvent set, spectrum, residual spectrum, range of $B \in L(\mathbb{X})$, respectively. It is well known that the operator $S$ defined as above is a contraction. Consider the quotient space $\mathbb{Y}:=l^{\infty}(\mathbb{X}) / c_{0}$ with the induced norm. The equivalent class containing a sequence $x \in l^{\infty}(\mathbb{X})$ will be denoted by $\bar{x}$. Since $S$ leaves $c_{0}$ invariant, it induces a bounded linear operator $\bar{S}$ acting in $\mathbb{Y}$. Moreover, one notes that $\bar{S}$ is a surjective isometry. As a consequence, $\sigma(\bar{S}) \subset \Gamma$, where $\Gamma$ denotes the unit circle in the complex plane. We will use the following estimate for the resolvent of the isometry $\bar{S}$, whose proof can be easily obtained:

$$
\|R(\lambda, \bar{S})\| \leq \frac{1}{\| \lambda|-1|}, \quad \text { for all }|\lambda| \neq 1 .
$$

\section{MAIN RESUlTS}

2.1. Katznelson-Tzafriri theorem for individual orbits. Consider the difference equation (1.1) with $(y(n)) \in c_{0}$. A main result of this paper is the following.

Theorem 2.1. Let $B$ be any linear continuous operator acting in $\mathbb{X}$ such that $\sigma(B) \cap \Gamma \subset\{1\}$, and let $x:=(x(n))_{n=1}^{\infty}$ be a bounded solution of (1.1). Then,

$$
\lim _{n \rightarrow \infty}[x(n+1)-x(n)]=0 .
$$

The theorem is an immediate consequence of several lemmas that may be of independent interest.

Lemma 2.2. Assume that $\bar{x}$ is any point in $\mathbb{Y}$ and that the complex function $g(\lambda):=R(\lambda, \bar{S}) \bar{x}$ has the point $\lambda=\xi_{0} \in \Gamma$ as an isolated singular point. Then, $\xi_{0}$ is either a removable singular point or a pole of first order.

Proof. Without loss of generality we may assume that $\xi_{0}=1$. Consider $\lambda$ in a small neighborhood of 1 in the complex plane. We will express $\lambda=e^{z}$ with $|z|<\delta_{0}$. Choose a small $\delta_{0}>0$ such that if $|z|<\delta_{0}$, then

$$
\frac{1}{|1-| \lambda||} \leq \frac{2}{|\Re z|} \text {. }
$$


Notice that if $0<|z|<\delta_{0}$, then

$$
\|R(\lambda, \bar{S}) \bar{x}\| \leq \frac{1}{|1-| \lambda||}\|\bar{x}\| \leq \frac{2}{|\Re z|}\|\bar{x}\| .
$$

Set $f(z)=R\left(e^{z}, \bar{S}\right) \bar{x}$ with $|z|<\delta_{0}$. Since 1 is a singular point of $\|R(\lambda, \bar{S}) \bar{x}\|, 0$ is a singular point of $f(z)$ in $\left\{|z|<\delta_{0}\right\}$. For each $n \in \mathbb{Z}$ and $0<r<\delta_{0}$, we have

$$
\begin{aligned}
& \left\|\frac{1}{2 \pi i} \int_{|z|=r} z^{n}\left(1+\frac{z^{2}}{r^{2}}\right) f(z) d z\right\| \\
& \quad \leq \frac{1}{2 \pi} \int_{|z|=r}\left|z^{n}\left(1+\frac{z^{2}}{r^{2}}\right)\right| \cdot\|f(z)\| \cdot|d z| .
\end{aligned}
$$

If $z=r e^{i \varphi}$, where $\varphi$ is real, then one has

$$
\begin{aligned}
\left|z^{n}\left(1+\frac{z^{2}}{r^{2}}\right)\right| & =r^{n}\left|1+e^{2 i \varphi}\right|=r^{n}\left|e^{-i \varphi}+e^{i \varphi}\right| \\
& =r^{n} 2|\cos \varphi|=2 r^{n-1}|\Re z| .
\end{aligned}
$$

Therefore,

$$
\begin{aligned}
\left|\frac{1}{2 \pi i} \int_{|z|=r} z^{n}\left(1+\frac{z^{2}}{r^{2}}\right) f(z) d z\right| & \leq \frac{1}{2 \pi} \int_{|z|=r} 2 r^{n-1}|\Re z| \frac{2}{|\Re z|} \cdot|d z| \\
& =\frac{2 \cdot 2 r^{n-1}}{2 \pi} \int_{|z|=r}|d z| \\
& =4 r^{n} .
\end{aligned}
$$

Consider the Laurent series of $f(z)$ at $z=0$,

$$
f(z)=\sum_{n=-\infty}^{\infty} a_{n} z^{n}
$$

where

$$
a_{n}=\frac{1}{2 \pi i} \int_{|z|=r} \frac{f(z) d z}{z^{n+1}}, \quad n \in \mathbb{Z}
$$

From (2.5) it follows that

$$
\begin{aligned}
\left\|a_{-(n+1)}+r^{-2} a_{-(n+3)}\right\| & =\left\|\frac{1}{2 \pi i} \int_{|z|=r} z^{n} f(z) d z+\frac{1}{2 \pi i} \int_{|z|=r} \frac{z^{n+2}}{r^{2}} f(z) d z\right\| \\
& =\left\|\frac{1}{2 \pi i} \int_{|z|=r} z^{n}\left(1+\frac{z^{2}}{r^{2}}\right) f(z) d z\right\| \\
& \leq 4 r^{n} .
\end{aligned}
$$

Therefore,

$$
\left\|r^{2} a_{-(n+1)}+a_{-(n+3)}\right\| \leq 4 r^{n+2}, \quad n \in \mathbb{Z} .
$$

Letting $r$ tend to 0 in (2.8), we come up with $a_{-k}=0$ for all $k \geq 2$. This shows that $z=0$ is a removable singular point (when $a_{-1}=0$ ) or a pole of first order of $f(z)$. This yields that the complex function $g(\lambda):=R(\lambda, \bar{S}) \bar{x}$ has $\lambda=1$ as a removable singular point or a pole of first order. The lemma is proven. 
Before proceeding we introduce some new notation: let $0 \neq z \in \mathbb{C}$ such that $z=r e^{i \varphi}$ with reals $r=|z|, \varphi$, and let $F(z)$ be any complex function. Then (with $s$ larger than $r$ ) we define

$$
\lim _{\lambda \downarrow z} F(\lambda):=\lim _{s \downarrow r} F\left(s e^{i \varphi}\right) .
$$

That is, we consider the limit as $\lambda$ approaches $z$ in a special direction corresponding to the ray $\arg \lambda=\arg z$.

Lemma 2.3. Let $\xi_{0} \in \Gamma$ be an isolated singular point of $g(\lambda)=R(\lambda, \bar{S}) \bar{x}$ with a given $\bar{x} \in \mathbb{Y}$. Then, this singular point $\xi_{0}$ is removable provided that

$$
\lim _{\lambda \downarrow \xi_{0}}\left(\lambda-\xi_{0}\right) R(\lambda, \bar{S}) \bar{x}=0 .
$$

Proof. As shown in Lemma 2.2, $\xi$ is either a removable singular point or a pole of first order. Without loss of generality we may assume that $\xi_{0}=1$ for the reader's convenience. Then, the Laurent series of $g(\lambda)$ is of the form

$$
g(\lambda)=\sum_{n=0}^{\infty}(\lambda-1)^{n} b_{n}+\frac{1}{\lambda-1} b_{-1} .
$$

We need to show that under condition (2.10) the coefficient $b_{-1}=0$. In fact,

$$
\begin{aligned}
0=\lim _{\lambda \downarrow 1} g(\lambda) & =\lim _{\lambda \downarrow 1}(\lambda-1) R(\lambda, \bar{S}) \bar{x} \\
& =\lim _{\lambda \downarrow 1}(\lambda-1)\left(\sum_{n=0}^{\infty}(\lambda-1)^{n} b_{n}+\frac{1}{\lambda-1} b_{-1}\right) \\
& =\lim _{\lambda \downarrow 1}\left(\sum_{n=0}^{\infty}(\lambda-1)^{n+1} b_{n}+\frac{\lambda-1}{\lambda-1} b_{-1}\right) \\
& =b_{-1} .
\end{aligned}
$$

This shows $\xi_{0}$ is removable. The lemma is proven.

Definition 2.4. Let $(x(n))$ be a bounded sequence in $\mathbb{X}$. The notation $\sigma(x)$ stands for the set of all non-removable singular points of the complex function $g(\lambda):=$ $R(\lambda, \bar{S}) \bar{x}$. This set may be referred to as the spectrum of $x$, an analog of a similar concept in [3]. Obviously, $\sigma(x)$ is a closed subset of $\Gamma$.

Lemma 2.5. Let $x:=(x(n))$ be a bounded solution of equation (1.1). Then,

$$
\sigma(x) \subset \sigma(B) \cap \Gamma \text {. }
$$

Proof. Consider $R(\lambda, \bar{S}) \bar{x}$ for all $|\lambda| \neq 1$. Since $x$ is a bounded solution of (1.1) and $\bar{y}=0$ we have

$$
\begin{aligned}
R(\lambda, \bar{S}) \bar{S} \bar{x} & =R(\lambda, \bar{S}) \bar{B} \bar{x}+R(\lambda, \bar{S}) \bar{y} \\
& =\bar{B} R(\lambda, \bar{S}) \bar{x} .
\end{aligned}
$$

On the other hand, the identity $\lambda R(\lambda, \bar{S}) \bar{x}-\bar{x}=R(\lambda, \bar{S}) \bar{S} \bar{x}$ gives

$$
\lambda R(\lambda, \bar{S}) \bar{x}-\bar{x}=R(\lambda, \bar{S}) \bar{S} \bar{x}=\bar{B} R(\lambda, \bar{S}) \bar{x},
$$

so

$$
\begin{aligned}
\bar{x} & =\lambda R(\lambda, \bar{S}) \bar{x}-\bar{B} R(\lambda, \bar{S}) \bar{x} \\
& =(\lambda-\bar{B}) R(\lambda, \bar{S}) \bar{x} .
\end{aligned}
$$


Obviously, $R(\lambda, \bar{S}) \bar{x}$ is analytic on $\mathbb{C} \backslash \Gamma$. Moreover, if $\left|\xi_{1}\right|=1$ and $\xi_{1} \notin \sigma(B) \cap \Gamma$ (as we can easily check that $\sigma(\bar{B})=\sigma(B)$ ), in a small neighborhood $U\left(\xi_{1}\right)$ of $\xi_{1}$ we have

$$
R(\lambda, \bar{S}) \bar{x} \quad=(\lambda-\bar{B})^{-1} \bar{x}, \quad \lambda \in U\left(\xi_{1}\right) \backslash \Gamma .
$$

This shows that $g(\lambda)=R(\lambda, \bar{S}) \bar{x}$ is analytically extendable to a neighborhood of $\xi_{1}$, that is, $\xi_{1} \notin \sigma(x)$. The lemma is proven.

Proof of Theorem 2.1. The identity $R(\lambda, \bar{S}) \bar{S} \bar{x}=\lambda R(\lambda, \bar{S}) \bar{x}-\bar{x}$ gives

$$
\begin{aligned}
R(\lambda, \bar{S})(\bar{S} \bar{x}-\bar{x}) & =R(\lambda, \bar{S}) \bar{S} \bar{x}-R(\lambda, \bar{S}) \bar{x} \\
& =\lambda R(\lambda, \bar{S}) \bar{x}-\bar{x}-R(\lambda, \bar{S}) \bar{x} \\
& =(\lambda-1) R(\lambda, \bar{S}) \bar{x}-\bar{x} .
\end{aligned}
$$

Therefore,

$$
h(\lambda):=(\lambda-1) R(\lambda, \bar{S})(\bar{S} \bar{x}-\bar{x})=(\lambda-1)^{2} R(\lambda, \bar{S}) \bar{x}-(\lambda-1) \bar{x} .
$$

By Lemmas 2.5 and 2.2, $\sigma(S x-x) \subset \sigma(B) \cap \Gamma \subset\{1\}, h(\lambda)$ is extendable analytically to the whole complex plane with the only possible exception at 1 . Since $g(\lambda):=$ $R(\lambda, \bar{S}) \bar{x}$ has 1 as either a removable singular point or a pole of first order, we have

$$
\lim _{\lambda \rightarrow 1}(\lambda-1)^{2} R(\lambda, \bar{S}) \bar{x}=0 .
$$

Consequently,

$$
\begin{aligned}
\lim _{\lambda \rightarrow 1}(\lambda-1) R(\lambda, \bar{S})(\bar{S} \bar{x}-\bar{x}) & =\lim _{\lambda \rightarrow 1}\left[(\lambda-1)^{2} R(\lambda, \bar{S}) \bar{x}-(\lambda-1) \bar{x}\right] \\
& =\lim _{\lambda \rightarrow 1}(\lambda-1)^{2} R(\lambda, \bar{S}) \bar{x}-\lim _{\lambda \rightarrow 1}(\lambda-1) \bar{x} \\
& =0 .
\end{aligned}
$$

By Lemma 2.3 $h(\lambda)$ has $\lambda=1$ as a removable singular point, so $h(\lambda)$ is extendable to an entire function. For $|\lambda|>1$, by (1.2) we have

$$
\begin{aligned}
\limsup _{|\lambda| \rightarrow \infty}\|h(\lambda)\| & =\limsup _{|\lambda| \rightarrow \infty}\|(\lambda-1) R(\lambda, \bar{S})(\bar{S} \bar{x}-\bar{x})\| \\
& \leq \limsup _{|\lambda| \rightarrow \infty} \frac{|\lambda|+1}{|\lambda|-1} \cdot\|\bar{S} \bar{x}-\bar{x}\| \\
& =\|\bar{S} \bar{x}-\bar{x}\| .
\end{aligned}
$$

This shows that $h(\lambda)$ is bounded on the complex plane, so as a bounded entire function it should be a constant by Liouville's Theorem. In turn, it is identically equal to zero because $h(1):=\lim _{\lambda \rightarrow 1} h(\lambda)=0$. Since $R(\lambda, \bar{S})$ is injective for each $\lambda \neq 1$, we have $\bar{S} \bar{x}-\bar{x}=0$. Therefore, $(S x-x) \in c_{0}$, that is, (2.1). The theorem is proven.

Remark 2.6. In the remark following Theorem 2.8 we will give an alternative proof of Theorem 2.1 in a more general context. However, the above proof seems to be more elementary. 
2.2. Stability of individual orbits. We define $\mathcal{M}_{\bar{x}}$ as the smallest closed subspace of $\mathbb{Y}:=l^{\infty}(\mathbb{X}) / c_{0}$ spanned by $\left\{\bar{S}^{n} \bar{x}, n \in \mathbb{Z}\right\}$. Consider the restriction $\left.\bar{S}\right|_{\mathcal{M}_{\bar{x}}}$ that is also a surjective isometry.

Lemma 2.7. Let $x:=(x(n)) \in l^{\infty}(\mathbb{X})$. Then, the following assertions hold:

i) $\sigma(x)=\emptyset$ if and only if $x \in c_{0}$;

ii) if $\sigma(\bar{x}) \neq \emptyset$, then $\sigma(x)=\sigma\left(\left.\bar{S}\right|_{\mathcal{M}_{\bar{x}}}\right)$.

Proof. (i) If $\sigma(x)=\emptyset$, the function $g(\lambda):=R(\lambda, \bar{S}) \bar{x}$ can be extended to an entire function. Using exactly the argument in the proof of Theorem 2.1 we come up with the boundedness of the complex function $t(\lambda):=(\lambda-1) R(\lambda, \bar{S}) \bar{x}$, so by Liouville's Theorem, $t(\lambda)$ is a constant. Thus, $t(\lambda)=\lim _{\lambda \rightarrow 1}(\lambda-1) g(\lambda)=0$. The injectiveness of $R(\lambda, \bar{S})$ for each $|\lambda| \neq 1$ yields that $\bar{x}=0$. The converse is clear.

(ii) By (i), $\bar{x} \neq 0$, so $\rho\left(\left.\bar{S}\right|_{\mathcal{M}_{\bar{x}}}\right) \neq \emptyset$. Let $\xi_{0} \in \rho\left(\left.\bar{S}\right|_{\mathcal{M}_{\bar{x}}}\right)$. Then, since for $|\lambda| \neq 1$,

$$
R(\lambda, \bar{S}) \bar{x}=R\left(\lambda,\left.\bar{S}\right|_{\mathcal{M}_{\bar{x}}}\right) \bar{x},
$$

it is clear that $\xi_{0}$ is a regular point of $g(\lambda)$.

Conversely, let $\xi_{0}$ be a regular point of $g(\lambda)$. Without loss of generality we may assume $\left|\xi_{0}\right|=1$; otherwise it is already in $\rho\left(\left.\bar{S}\right|_{\mathcal{M}_{\bar{x}}}\right)$. We will show that $\xi_{0} \in \rho\left(\left.\bar{S}\right|_{\mathcal{M}_{\bar{x}}}\right)$ by proving that the equation

$$
\xi_{0} v-\bar{S} v=w
$$

has a unique solution $v \in \mathcal{M}_{\bar{x}}$ for each given $w \in \mathcal{M}_{\bar{x}}$. First, we show that there is at least one solution. In fact, we note that for each $n \in \mathbb{Z}$ the set of regular points of $g(\lambda)=R(\lambda, \bar{S}) \bar{x}$ is the same as that of $\bar{S}^{n} g(\lambda)=R(\lambda, \bar{S}) \bar{S}^{n} \bar{x}$. In turn, by the property of holomorphic functions, the set of all regular points of $g(\lambda)=R(\lambda, \bar{S}) \bar{x}$ must be part of that of the function $k(\lambda)=R(\lambda, \bar{S}) w$, so $k(\lambda)=R(\lambda, \bar{S}) w$ is analytically extendable to a neighborhood of $\xi_{0}$. In particular, $\lim _{\lambda \rightarrow \xi_{0}} k(\lambda)=v \in \mathcal{M}_{\bar{x}}$, so

$$
\begin{aligned}
\lim _{(|\lambda|>1), \lambda \rightarrow \xi_{0}}[\lambda R(\lambda, \bar{S}) w-R(\lambda, \bar{S}) \bar{S} w] & =w \\
\xi_{0} v-\bar{S} v & =w .
\end{aligned}
$$

To show that equation (2.17) has a unique solution in $\mathcal{M}_{\bar{x}}$ we can show that the homogeneous equation $\xi_{0} v-\bar{S} v=0$ has only a trivial solution in $\mathcal{M}_{\bar{x}}$. In fact, let $v_{0} \in \mathcal{M}_{\bar{x}}$ be a solution of this equation. Then, for each $|\lambda|>1$, using the identity $R(1, A)=(I-A)^{-1}=\sum_{n=0}^{\infty} A^{n}$ for each $\|A\|<1$ and $\bar{S}^{n} v_{0}=\xi_{0}^{n} v_{0}$, we have

$$
\begin{aligned}
R(\lambda, \bar{S}) v_{0} & =\sum_{n=0}^{\infty} \frac{1}{\lambda^{n+1}} \bar{S}^{n} v_{0} \\
& =\sum_{n=0}^{\infty} \frac{1}{\lambda^{n+1}} \xi_{0}^{n} v_{0} \\
& =\frac{1}{\lambda-\xi_{0}} v_{0} .
\end{aligned}
$$

Since $v_{0} \in \mathcal{M}_{\bar{x}}$, this function must, as above, be extendable analytically to a neighborhood of $\xi_{0}$, and this is possible only if $v_{0}=0$. Summing up, we have that $\xi_{0} \in \rho\left(\mathcal{M}_{\bar{x}}\right)$, so the lemma is proven. 
Theorem 2.8. Let $(x(n))$ be a bounded sequence such that the set $\sigma(x)$ of all nonremovable singular points of $g(\lambda)=R(\lambda, \bar{S}) \bar{x}$ is countable, and let the following condition hold for each $\xi_{0} \in \sigma(\bar{x})$ :

$$
\lim _{\lambda \downarrow \xi_{0}}\left(\lambda-\xi_{0}\right) R(\lambda, \bar{S}) \bar{x}=0 .
$$

Then,

$$
\lim _{n \rightarrow \infty} x(n)=0 .
$$

Proof. We have to show (2.20); that is, $\bar{x}=0$, or equivalently, $\mathcal{M}_{\bar{x}}$ is trivial. Suppose to the contrary that it is not. Then, by Lemma 2.7, $\sigma(x)=\sigma\left(\left.\bar{S}\right|_{\mathcal{M}_{\bar{x}}}\right) \neq \emptyset$. Since $\sigma(x)$ is a non-empty closed subset of $\Gamma$ and is countable, it has an isolated point, say $\xi_{0}$, so $\xi_{0}$ is an isolated singular point for $g(\lambda)$. By Lemma2.2 this isolated singular point must be either a removable singular point or a pole of first order. Since $\xi_{0}$ is a pole of first order of the resolvent $R\left(\lambda,\left.\bar{S}\right|_{\mathcal{M}_{\bar{x}}}\right)$, by a well-known result in Functional Analysis 1 (see e.g. [18, Theorem 5.8 A, p. 306] or [22, Theorem 3, p. 229]), $\xi_{0}$ must be an eigenvalue of $\left.\bar{S}\right|_{\mathcal{M}_{\bar{x}}}$ with a non-zero eigenvector $w_{0}$. As in the proof of Lemma 2.7 (see 2.18) ), for each $|\lambda| \neq 1$ we have

$$
R(\lambda, \bar{S}) w_{0}=\frac{1}{\lambda-\xi_{0}} w_{0} .
$$

On the other hand, by Lemma 2.3, $\xi_{0}$ is a removable singular point for $g(\lambda)$, so is for $R(\lambda, \bar{S}) w_{0}$. This is possible only if $w_{0}=0$, contradicting that $w_{0}$ is a non-zero vector. This proves the theorem.

Remark 2.9. An alternative proof of Theorem 2.1 is a direct application of Lemma 2.5 and Theorem 2.8. As another consequence of Theorem 2.8 we have the following on the strong asymptotical stability of solutions of (1.1).

Theorem 2.10. For equation (1.1) assume that $(y(n)) \in c_{0}$ and that the operator $B$ in equation (1.1) has $\sigma(B) \cap \Gamma$ as a countable set. Then, the following holds for each bounded solution $(x(n))$ of (1.1):

$$
\lim _{n \rightarrow \infty} x(n)=0,
$$

provided that for each $\xi_{0} \in \sigma(B) \cap \Gamma$ the following condition holds:

$$
\lim _{\lambda \downarrow \xi_{0}}\left(\lambda-\xi_{0}\right) R(\lambda, \bar{S}) \bar{x}=0 .
$$

Proof. This theorem is an immediate consequence of Lemma 2.5] and Theorem 2.8,

\section{Discussion}

Theorem 2.1 may be seen as an extension of the following result of KatznelsonTzafriri (see [12, Theorem 1]).

Theorem 3.1. Let $T$ be a power bounded linear operator in a Banach space $\mathbb{X}$ such that $\sigma(T) \cap \Gamma \subset\{1\}$. Then,

$$
\lim _{n \rightarrow \infty}\left(T^{n+1}-T^{n}\right)=0 .
$$

In fact, as noted in [20, this theorem is equivalent to a weaker one.

\footnotetext{
${ }^{1}$ We actually avoid applying the Gel'fand Theorem in this case.
} 
Theorem 3.2. Let $T$ be a power bounded linear operator in a Banach space $\mathbb{X}$ such that $\sigma(T) \cap \Gamma \subset\{1\}$. Then, for each $x_{0} \in \mathbb{X}$,

$$
\lim _{n \rightarrow \infty}\left(T^{n+1} x_{0}-T^{n} x_{0}\right)=0 .
$$

Obviously, our Theorem 2.1 extends Theorem 3.2

As an immediate consequence of Theorem 2.10 we have the following corollary:

Corollary 3.3. Let $B \in L(\mathbb{X})$ be a power bounded operator such that $\sigma(B) \cap \Gamma$ is a countable set. Moreover, assume that for each $\xi_{0} \in \sigma(B) \cap \Gamma$ the following holds for each $x_{0} \in \mathbb{X}$ :

$$
\lim _{\lambda \downarrow \xi_{0}}\left(\lambda-\xi_{0}\right) R(\lambda, B) x_{0}=0 .
$$

Then, for every $x_{0} \in \mathbb{X}$,

$$
\lim _{n \rightarrow \infty} B^{n} x_{0}=0 .
$$

Proof. Let $x(n)=B^{n} x_{0}$. Then, $(x(n))$ is a bounded solution of (1.1) with $(y(n))=$ 0 . Therefore, if $|\lambda|>1, \lambda \in \rho(B)$ and $\lambda \in \rho(\bar{S})$, so by (2.15) (and the proof of Lemma 2.5),

$$
\begin{aligned}
\lim _{\lambda \downarrow \xi_{0}}\left\|\left(\lambda-\xi_{0}\right) R(\lambda, \bar{S}) \bar{x}\right\| & =\lim _{\lambda \downarrow \xi_{0}}\left\|\left(\lambda-\xi_{0}\right) R(\lambda, \bar{B}) \bar{x}\right\| \\
& \leq \lim _{\lambda \downarrow \xi_{0}} \sup _{n \in \mathbb{N}}\left\{\left\|\left(\lambda-\xi_{0}\right) R(\lambda, B) B^{n} x_{0}\right\|\right\} \\
& \leq \lim _{\lambda \downarrow \xi_{0}} \sup _{n \in \mathbb{N}}\left\{\left\|B^{n}\right\|\right\} \cdot\left\|\left(\lambda-\xi_{0}\right) R(\lambda, B) x_{0}\right\| \\
& \leq \lim _{\lambda \downarrow \xi_{0}}\left\|\left(\lambda-\xi_{0}\right) R(\lambda, B) x_{0}\right\|=0 .
\end{aligned}
$$

Therefore, by Theorem 2.10, $x(n)=B^{n} x_{0} \rightarrow 0$.

Remark 3.4. Condition (3.3) is satisfied if $\operatorname{R\sigma }(B) \cap \Gamma=\emptyset$, and hence Corollary 3.3 yields the discrete version of the Arendt-Batty-Lyubich-Vu Theorem [2, Theorem 5.1], [10, Corollary 3.3], 21]). In fact, since $B$ is power-bounded one can easily show that there exists a positive constant $C$ such that

$$
\|R(\lambda, B)\| \leq \frac{C}{|| \lambda|-1|}, \quad \text { for }|\lambda|>1
$$

Next, since $R \sigma(B) \cap \Gamma=\emptyset$, for all $\xi_{0} \in \sigma(B) \cap \Gamma$, the range of $\left(\xi_{0}-B\right)$ is dense in $\mathbb{X}$. Therefore, for each $x_{0} \subset \mathbb{X}$ there is a sequence $\left(x_{0}^{n}\right) \in \operatorname{Ran}\left(\xi_{0}-B\right)$ such that $x_{0}=\lim _{n \rightarrow \infty} x_{0}^{n}$. Then, $x_{0}^{n}=\left(\xi_{0}-B\right) y_{0}^{n}$ for some sequence $\left(y_{0}^{n}\right) \subset \mathbb{X}$. By our definition of the limit as $\lambda \downarrow \xi_{0}$ we have $\left|\lambda-\xi_{0}\right|=|| \lambda|-| \xi_{0}||=|| \lambda|-1| \rightarrow 0$, so in 
view of (3.7), for each fixed $n$ we have

$$
\begin{aligned}
\lim _{\lambda \downarrow \xi_{0}}\left\|\left(\lambda-\xi_{0}\right) R(\lambda, B) x_{0}^{n}\right\| & =\lim _{\lambda \downarrow \xi_{0}}\left\|\left(\lambda-\xi_{0}\right) R(\lambda, B)\left(\xi_{0}-B\right) y_{0}^{n}\right\| \\
& =\lim _{\lambda \downarrow \xi_{0}}\left\|\left(\lambda-\xi_{0}\right) R(\lambda, B)\left[(\lambda-B) y_{0}^{n}+\left(\xi_{0}-\lambda\right) y_{0}^{n}\right]\right\| \\
& =\lim _{\lambda \downarrow \xi_{0}}\left(\left|\lambda-\xi_{0}\right| \cdot\left\|y_{0}^{n}\right\|+\left\|\left(\lambda-\xi_{0}\right) R(\lambda, B)\left(\xi_{0}-\lambda\right) y_{0}^{n}\right\|\right) \\
& \leq 0+\lim _{\lambda \downarrow \xi_{0}}\left\|\lambda-\xi_{0}\right\|^{2} \cdot\|R(\lambda, B)\| \cdot\left\|y_{0}^{n}\right\| \\
& \leq \lim _{\lambda \downarrow \xi_{0}}\left\|\lambda|-| \xi_{0}\right\|^{2} \cdot \frac{C}{\| \lambda|-1|} \cdot\left\|y_{0}^{n}\right\| \\
& =\lim _{\lambda \downarrow \xi_{0}} \frac{\| \lambda|-1|^{2} \cdot C}{\| \lambda|-1|} \cdot\left\|y_{0}^{n}\right\|=0 .
\end{aligned}
$$

By (3.7) for every fixed $n$,

$$
\limsup _{\lambda \downarrow \xi_{0}}\left\|\left(\lambda-\xi_{0}\right) R(\lambda, B)\left(x_{0}-x_{0}^{n}\right)\right\| \leq C\left\|x_{0}-x_{0}^{n}\right\| .
$$

Finally, for each $n \in \mathbb{N}$ from (3.8) and (3.9) we have

$$
\begin{aligned}
\limsup _{\lambda \downarrow \xi_{0}}\left\|\left(\lambda-\xi_{0}\right) R(\lambda, B) x_{0}\right\| \leq & \limsup _{\lambda \downarrow \xi_{0}}\left[\|\left(\lambda-\xi_{0}\right) R(\lambda, B) x_{0}^{n}\right. \\
& \left.+\left(\lambda-\xi_{0}\right) R(\lambda, B)\left(x_{0}-x_{0}^{n}\right) \|\right] \\
\leq & C\left\|x_{0}-x_{0}^{n}\right\| .
\end{aligned}
$$

Since $\left\|x_{0}^{n}-x_{0}\right\| \rightarrow 0$ as $n \rightarrow \infty$, we have that (3.3) holds for any $x_{0} \in \mathbb{X}$.

Let us define a so-called Condition $H$ for a closed subspace $\mathcal{M}$ of $l^{\infty}(\mathbb{X})$ by the following axioms:

i) $\mathcal{M}$ is bi-invariant under translation $S$, that is, $\mathcal{M}=\left\{x \in l^{\infty}(\mathbb{X}): S x \in \mathcal{M}\right\}$;

ii) if $x:=(x(n)) \in \mathcal{M}$ and $A \in L(\mathbb{X})$, then $y:=(A x(n)) \in \mathcal{M}$;

iii) $c_{0} \subset \mathcal{M}$.

As an example of such a closed subspace $\mathcal{M}$ of $l^{\infty}(\mathbb{X})$ that satisfies Condition $\mathrm{H}$ one can take the space $A A P(\mathbb{N}, \mathbb{X})$ of all asymptotic almost periodic sequences. If we replace $c_{0}$ by $\mathcal{M}$, we will arrive at various analogs of Theorems 2.1, 2.10 and 2.8. Note that the proofs of these analogs are identically similar to those of the mentioned theorems. Below are the statements of analogs of the mentioned theorems in the case $\mathcal{M}=A A P(\mathbb{N}, \mathbb{X})$.

Recall that a sequence $(x(n))$ is said to be asymptotically almost periodic if $x(n)=y(n)+z(n)$ for all $n \in \mathbb{N}$ where $(y(n)) \in c_{0}$ and $(z(n))$ is an almost periodic sequence. An almost periodic sequence on $\mathbb{N}$ is the restriction to $\mathbb{N}$ of an almost periodic sequence on $\mathbb{Z}$. In turn, an almost periodic sequence on $\mathbb{Z}$ is defined to be an element of the following subspace $\overline{\operatorname{span}\left\{\left(\lambda^{n} y_{0}\right)_{n \in \mathbb{Z}}, \lambda \in \Gamma, y_{0} \in \mathbb{X}\right\}}$ of $l^{\infty}(\mathbb{X})$. In the following, by abusing notation, $\bar{x}$ denotes the equivalence class of $l^{\infty}(\mathbb{X}) / A A P(\mathbb{N}, \mathbb{X})$ containing $x$, and $\bar{S}$ denotes the operator acting in $l^{\infty}(\mathbb{X}) / A A P(\mathbb{N}, \mathbb{X})$ induced by $S$.

Theorem 3.5. Let $B$ be any linear continuous operator acting in $\mathbb{X}$ such that $\sigma(B) \cap \Gamma \subset\{1\}$, and let $x:=(x(n))_{n=1}^{\infty}$ be a bounded solution of (1.1) in which $(y(n)) \in A A P(\mathbb{N}, \mathbb{X})$. Then, the sequence $(y(n))$, defined as $y(n):=x(n+1)-x(n)$ for all $n \in \mathbb{N}$, is asymptotically almost periodic. 
Theorem 3.6. Let $(x(n))$ be a bounded sequence such that the set $\sigma_{A A P(\mathbb{N}, \mathbb{X})}(x)$ of all non-removable singular points of $g(\lambda)=R(\lambda, \bar{S}) \bar{x}$ is countable, and let the following condition hold for each $\xi_{0} \in \sigma_{A A P(\mathbb{N}, \mathbb{X})}(x)$ :

$$
\lim _{\lambda \downarrow \xi_{0}}\left(\lambda-\xi_{0}\right) R(\lambda, \bar{S}) \bar{x}=0 .
$$

Then, $(x(n))$ is asymptotically almost periodic.

Theorem 3.7. For equation (1.1) assume that $(y(n)) \in A A P(\mathbb{N}, \mathbb{X})$, and the operator $B$ in equation (1.1) has $\sigma(B) \cap \Gamma$ as a countable set. Then, each bounded solution $(x(n))$ of (1.1) is asymptotically almost periodic, provided that for each $\xi_{0} \in \sigma(B) \cap \Gamma$ the following holds:

$$
\lim _{\lambda \downarrow \xi_{0}}\left(\lambda-\xi_{0}\right) R(\lambda, \bar{S}) \bar{x}=0 .
$$

\section{REFERENCES}

1. G. R. Allan, T. J. Ransford, Power-dominated elements in a Banach algebra. Studia Math. 94 (1989), 63-79. MR1008239 (91a:46050)

2. W. Arendt, C. J. K. Batty, Tauberian theorems and stability of one-parameter semigroups, Trans. Amer. Math. Soc. 306 (1988), 837-852. MR.933321 (89g:47053)

3. W. Arendt, C. J. K. Batty, Asymptotically almost periodic solutions of inhomogeneous Cauchy problems on the half-line. Bull. London Math. Soc. 31 (1999), 291-304. MR.1673408 (2001d:34085)

4. W. Arendt, C. J. K. Batty, M. Hieber, F. Neubrander, Vector-valued Laplace transforms and Cauchy problems, Monographs in Mathematics, 96, Birkhäuser Verlag, Basel, 2001. MR.1886588 (2003g:47072)

5. W. Arendt, J. Pruss, Vector-valued Tauberian theorems and asymptotic behavior of linear Volterra equations. SIAM J. Math. Anal. 23 (1992), 412-448. MR1147871 (92m:47150)

6. B. Basit, A. J. Pryde, Ergodicity and stability of orbits of unbounded semigroup representations. J. Aust. Math. Soc. 77 (2004), 209-232. MR2083746 (2005g:47073)

7. C. J. K. Batty, Jan van Neerven, Frank Rabiger, Local spectra and individual stability of uniformly bounded $C_{0}$-semigroups. Trans. Amer. Math. Soc. 350 (1998), 2071-2085. MR 1422890 (98h:47054)

8. R. Chill, Y. Tomilov, Stability of operator semigroups: ideas and results. In Perspectives in Operator Theory. Banach Center Publications, Vol. 75 (2007), pp. 71-109. MR2336713 (2008m:47054)

9. J. Esterle, E. Strouse, F. Zouakia, Stabilité asymptotique de certains semigroupes d'opérateurs et idéaux primaires de $L^{1}\left(\mathbb{R}_{+}\right)$. J. Operator Theory 28 (1992), 203-227. MR.1273043 (95f:43001)

10. J. Esterle, E. Strouse, F. Zouax, Theorems of Katznelson-Tzafriri type for contractions. J. Funct. Anal. 94 (1990), 273-287. MR1081645(92c:47016)

11. N. Kalton, S. Montgomery-Smith, K. Oleszkiewicz, Y. Tomilov, Power-bounded operators and related norm estimates. J. London Math. Soc. (2) 70 (2004), 463-478. MR2078905 (2005e:47020)

12. Y. Katznelson, L. Tzafriri, On power bounded operators. J. Funct. Anal. 68 (1986), 313-328. MR859138 (88e:47006)

13. R. deLaubenfels, Quôc Phong Vu, Stability and almost periodicity of solutions of ill-posed abstract Cauchy problems. Proceedings of the A.M.S. 125 (1997), 235-241. MR.1350938 (97c:34123)

14. Nguyen Van Minh, Katznelson-Tzafriri type theorems for individual solutions of evolution equations. Proceedings of the A.M.S. 136 (2008), 1749-1755. MR.2373605

15. Nguyen Van Minh, A new approach to the spectral theory of functions and the Loomis-Arendt-Batty-Vu Theory. Submitted. Preprint in arXiv.org at the URL: http://arxiv.org/abs/math.FA/0609652

16. H. Mustafayev, The Banach algebra generated by a $C_{0}$-semigroup. C. R. Math. Acad. Sci. Paris 342 (2006), no. 8, 575-578. MR2217918 (2007a:47050) 
17. J. M. A. M. van Neerven, The asymptotic behaviour of semigroups of linear operators, Birkäuser Verlag, Basel-Boston-Berlin, Operator Theory, Advances and Applications, Vol. 88, 1996. MR.1409370 (98d:47001)

18. A. E. Taylor, Introduction to Functional Analysis, John Wiley \& Sons, New York-London, 1958. MR0098966 (20:5411)

19. Quoc Phong Vu, Theorems of Katznelson-Tzafriri type for semigroups of operators. J. Funct. Anal. 103 (1992), 74-84. MR.1144683 (93e:47050)

20. Quoc Phong Vu, A short proof of Y. Katznelson's and L. Tzafriri's theorem. Proceedings of the A.M.S., 115 (1992), pp. 1023-1024. MR1087468 (92j:47012)

21. Quoc Phong Vu, Yu. I. Lyubich, A spectral criterion for almost periodicity for one-parameter semigroups (Russian). Teor. Funktsii Funktsional. Anal. i Prilozhen. No. 47 (1987), 36-41; translation in J. Soviet Math. 48 (1990), no. 6, 644-647. MR916441 (89a:47067)

22. K. Yosida, Functional Analysis, fifth edition, Springer, Berlin-Heidelberg-New York, 1978. MR0500055 (58:17765)

Department of Mathematics, University of West Georgia, Carrollton, Georgia 30118

E-mail address: vnguyen@westga.edu 